\title{
An Extremely Rare Source of Pulmonary Embolism: Internal Jugular Vein Aneurysm
}

\author{
Utkan Sevuk $^{\mathrm{a}, \mathrm{d}}$, Firat Ayaz ${ }^{\mathrm{a}}$, Ertan Demirdas ${ }^{\mathrm{b}}$, Aylin Erkul $^{\mathrm{c}}$
}

\begin{abstract}
Jugular venous aneurysm (JVA) is a rare venous anomaly that usually presents in children and is infrequently reported in the adult literature. We present a case of an internal jugular vein aneurysm (IJVA) in an adult patient who presented with pulmonary embolism. A 70-yearold female was admitted to the hospital with pulmonary embolism. A left IJVA was found incidentally which was $22 \times 18 \mathrm{~mm}$ in diameter during the screening for malignancy. Duplex ultrasound revealed residual venous thrombosis. The patient had no other risk factors for pulmonary embolism. Although extremely rare, IJVA may be a causative factor of pulmonary embolism.
\end{abstract}

Keywords: Pulmonary embolism; Internal jugular vein aneurysm; Adult

\section{Introduction}

Jugular venous aneurysm (JVA) is a rare venous anomaly that usually presents in children and is infrequently reported in the adult literature [1]. Most patients present with an asymptomatic neck mass that appears while coughing, straining, bending, or breath holding. We present a case of an internal jugular vein aneurysm (IJVA) in an adult patient who presented with pulmonary embolism. As far as we know, this is the first report of pulmonary embolism in an adult patient with IJVA.

\section{Case Report}

Institutional review board approval was obtained for the de-

\footnotetext{
Manuscript accepted for publication April 18, 2016

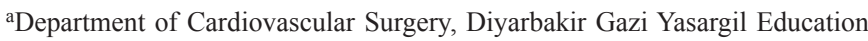
and Research Hospital, Diyarbakir, Turkey

${ }^{b}$ Department of Cardiovascular Surgery, Bozok University, Yozgat, Turkey ${ }^{\mathrm{c}}$ Department of Anesthesiology, Diyarbakir Obstetrics and Gynecology Hospital, Diyarbakir, Turkey

${ }^{\mathrm{d} C}$ Corresponding Author: Utkan Sevuk, Diyarbakir Gazi Yasargil Egitim ve Arastirma Hastanesi, Kalp ve Damar Cerrahisi Klinigi, 3. kat, Uckuyular, Diyarbakir, Turkey. Email: utkansevuk@gmail.com
}

doi: http://dx.doi.org/10.14740/jmc2485w scribed case. A 70-year-old female presented to the emergency department of our institution with a 12-h history of sudden onset breathlessness and persistent right-sided non-radiating chest pain. She had no history of smoking, malignancy, inflamatuar disease, venous thromboembolism, surgery or hormone replacement therapy and hereditary thrombophilia and no significant family history of any medical illness. There was also no history of shortness of breath or any recent long-distance travel. The patient's body mass index was 25 . Electrocardiogram obtained on admission to hospital revealed normal sinus rhythm with a rate of $86 \mathrm{bpm}$. Transthoracic echocardiogram was negative for thrombus with normal ventricular dimensions and functions. A D-dimer assay was taken and found to be raised. Therefore, contrast-enhanced computed tomography (CECT) scan of the chest was carried out which revealed intraluminal filling defects in the right pulmonary artery (Fig. 1). Further testing was done following hospitalization to determine the etiology. Blood tests demonstrated normal full blood count and biochemistry. Thrombophilia test results (factor V Leiden, prothrombin gene mutation, antithrombin, protein $\mathrm{C}$ and protein $\mathrm{S}$, and lupus anticoagulant) were negative. Deep venous thrombosis of the extremities was excluded with duplex scan. Diagnostic screening workup for occult malignancy (cervical, cranial and abdominopelvic CT) was negative. On the other hand, a left IJVA was found incidentally which was $22 \times 18 \mathrm{~mm}$ in diameter (Fig. 2). Duplex ultrasound revealed residual thrombus adherent to the vein wall. The patient re-

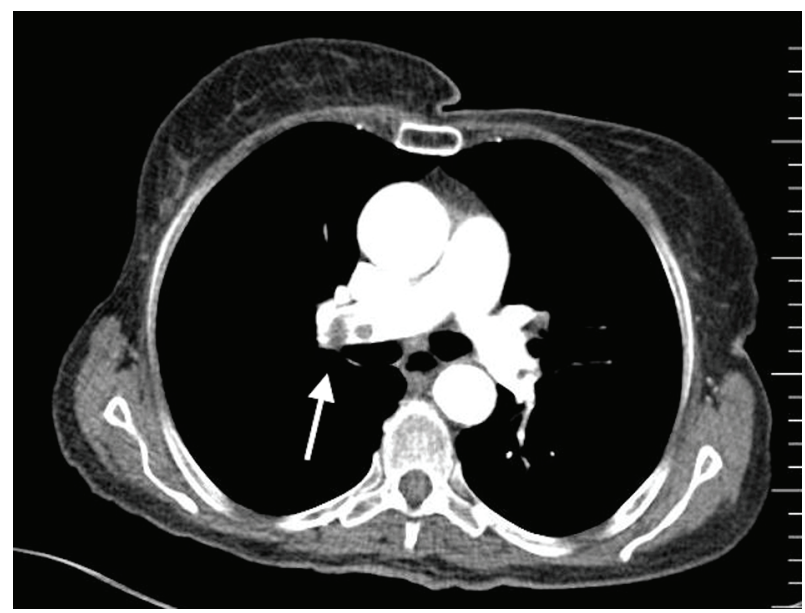

Figure 1. Computed tomography scan shows the thrombus within the right main pulmonary artery (white arrow). 


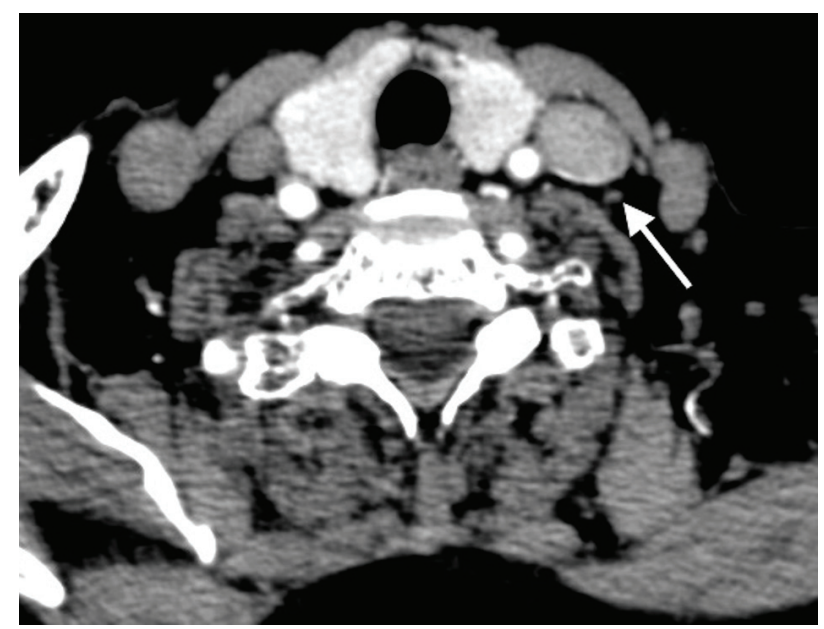

Figure 2. Computed tomography scan demonstrates the internal jugular vein aneurysm (white arrow).

fused surgical treatment and was anticoagulated with warfarin sodyum to target INR of 2.5 .

Pulmonary embolism recurrence has not occurred at a follow-up of 6 months.

\section{Discussion}

There have been sporadic reports only of JVA in the English literature. Due to its asymptomatic nature, JVA can be underlooked by the patient as well as the clinician. Thus, the incidence of pulmonary embolism may be underreported in patients with JVA.

JVA usually presents as a soft cystic mass in the neck that transiently appears on straining and frequently occurs on the right side. JVA can be complicated by thrombus, pulmonary embolism, spontaneous rupture and thrombophlebitis [2]. The differential diagnosis includes non-pulsatile soft neck masses that enlarge on the valsalva maneuver: laryngocele, cupular inflation, external laryngeal diverticula, pharyngocele, venolymphatic malformations and superior mediastinal tumor or cyst [2].

While the etiology of JVA is unknown, several etiologic hypotheses have been proposed in previous reports. Histological studies have revealed loss of the elastic layer and hypertrophy of the connective tissue, with focal intimal thickening [3]. Thus, a congenital structural defect in the vein wall may be an important factor in the development of JVA. Any defect of the venous valve placed at the entrance of the internal and external jugular veins, mechanical compression or trauma of the vein and conditions leading to obstruction of the jugular vein (such as thoracic outlet syndrome and anterior scalenus compression) and chronically increased intra-thoracic pressure may be other important causative factors [3].

Doppler ultrasound with valsalva maneuvre is the preferred initial study [4]. Other diagnostic modalities include CECT, CT angiography or MR venography [4]. CT and MR have the advantage of demonstrating the deep anatomical structures adjacent to the jugular vein.

Treatment options include resection, ligation, surgical resection with lateral venorrhaphy and conservative management $[1,2,5]$. The management of asymptomatic JVA is still controversial. While some authors advocate a conservative therapy, most of the authors recommend surgical treatment before a complication occurs. Surgical treatment is recommended in symptomatic patients and for cosmetic reasons [1,2]. Although jugular veins drain both cerebral hemispheres (approximately $70 \%$ ipsilateral and 30\% contralateral), right internal jugular vein (IJV) is dominant in majority of the patients. Given the potential risk of increased intracranial pressure due to venous congestion following ligation of the IJV, which may lead to vomiting, headache, ipsilateral neck and craniofacial swelling, and lacunar cerebral infarction, longitudinal constriction suture venoplasty plus encapsulation has been recommended instead of ligation and resection particularly in patients with right IJVA or bilateral IJVA [1, 2]. Endoscopic repair has also been reported in the literature [5].

Although extremely rare, IJVA may be a causative factor of pulmonary embolism. The clinical diagnosis of JVA begins with high index of suspicion.

\section{Conflicts of Interest}

The authors declare that there is no conflicts of interest.

\section{Author Contributions}

US, FA, and AE were involved in management of the patient. US and ED drafted the manuscript. FA and AE provided valuable input and guidance during the preparation of the manuscript. ED supervised the writing critically. All authors read and approved the final manuscript.

\section{References}

1. Calligaro KD, Ahmad S, Dandora R, Dougherty MJ, Savarese RP, Doerr KJ, McAffee S, et al. Venous aneurysms: surgical indications and review of the literature. Surgery. 1995;117(1):1-6.

2. Jianhong L, Xuewu J, Tingze H. Surgical treatment of jugular vein phlebectasia in children. Am J Surg. 2006;192(3):286-290.

3. Paleri V, Gopalakrishnan S. Jugular phlebectasia: theory of pathogenesis and review of literature. Int $\mathrm{J}$ Pediatr Otorhinolaryngol. 2001;57(2):155-159.

4. Bora MK. Internal Jugular Phlebectasia: Diagnosis by Ultrasonography, Doppler and Contrast CT. J Clin Diagn Res. 2013;7(6):1194-1196.

5. Chang YT, Lee JY, Wang JY, Chiou CS. Transaxillary subfascial endoscopic approach for internal jugular phlebectasia in a child. Head Neck. 2010;32(6):806-811. 